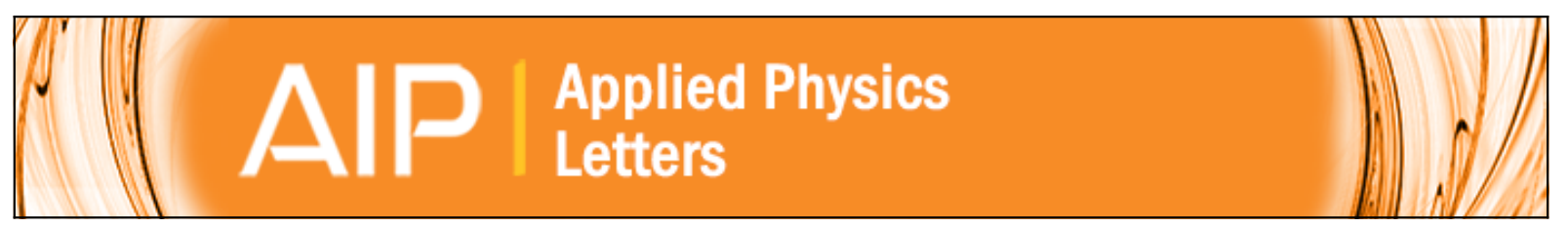

\title{
Determination of the electron-phonon coupling constant in tungsten
}

Szymon L. Daraszewicz, Yvelin Giret, Hiroshi Tanimura, Dorothy M. Duffy, Alexander L. Shluger, and Katsumi Tanimura

Citation: Applied Physics Letters 105, 023112 (2014); doi: 10.1063/1.4890413

View online: http://dx.doi.org/10.1063/1.4890413

View Table of Contents: http://scitation.aip.org/content/aip/journal/apl/105/2?ver=pdfcov

Published by the AIP Publishing

\section{Articles you may be interested in}

Orbital dependent Rashba splitting and electron-phonon coupling of 2D Bi phase on $\mathrm{Cu}(100)$ surface

J. Chem. Phys. 139, 184707 (2013); 10.1063/1.4828865

Electron-phonon scattering dynamics in ferromagnets on ultrafast timescales: Influence of the phonon temperature

J. Appl. Phys. 111, 07C514 (2012); 10.1063/1.3676611

Two-dimensional electron gases: Theory of ultrafast dynamics of electron-phonon interactions in graphene, surfaces, and quantum wells

J. Appl. Phys. 105, 122409 (2009); 10.1063/1.3117236

Phonon spectrum and electron-phonon interaction in technetium

Low Temp. Phys. 31, 449 (2005); 10.1063/1.1925373

Electron-phonon coupling dynamics in very small (between 2 and $8 \mathrm{~nm}$ diameter) Au nanoparticles

J. Chem. Phys. 112, 5942 (2000); 10.1063/1.481167

\section{AlP $\mid \begin{aligned} & \text { Journal of } \\ & \text { Applied Physics }\end{aligned}$}

Journal of Applied Physics is pleased to announce André Anders as its new Editor-in-Chief 


\title{
Determination of the electron-phonon coupling constant in tungsten
}

\author{
Szymon L. Daraszewicz, ${ }^{1}$ Yvelin Giret, ${ }^{1,2}$ Hiroshi Tanimura, ${ }^{2}$ Dorothy M. Duffy, ${ }^{1}$ \\ Alexander L. Shluger, ${ }^{1}$ and Katsumi Tanimura ${ }^{2}$ \\ ${ }^{1}$ Department of Physics and Astronomy, London Centre for Nanotechnology, University College London, \\ Gower Street, WCIE 6BT London, United Kingdom \\ ${ }^{2}$ The Institute of Scientific and Industrial Research (ISIR), Osaka University, Mihogaoka 8-1, Ibaraki, \\ Osaka 567-0047, Japan
}

(Received 21 May 2014; accepted 27 June 2014; published online 18 July 2014)

\begin{abstract}
We used two methods to determine the effective electron-phonon coupling constant $\left(G_{0}\right)$ in tungsten. Our first principles calculations predict $G_{0}=1.65 \times 10^{17} \mathrm{~W} \mathrm{~m}^{-3} \mathrm{~K}^{-1}$. The temporal decay of the femtosecond-resolution optical reflectivity for a (100) surface of bulk W was measured using a pump-probe scheme and analysed using ab initio parameterised two temperature model, which includes both the effects of the electron-phonon coupling and thermal conduction into bulk. This analysis gives $G_{0}=1.4(3) \times 10^{17} \mathrm{~W} \mathrm{~m}^{-3} \mathrm{~K}^{-1}$, in good agreement with the theoretical prediction. The described effective method of calculating and measuring $G_{0}$ in bulk materials can be easily extended to other metals. (C) 2014 AIP Publishing LLC. [http://dx.doi.org/10.1063/1.4890413]
\end{abstract}

The effective strength of the electron-phonon (e-p) interaction, $G_{0}$, controls the energy transfer between electrons and ions and is essential for understanding the behavior of a material in an electron-ion non-equilibrium state. The e-p coupling enters into a variety of non-equilibrium dynamics models based on the two temperature (2T) model ${ }^{1,2}$ and is also central to the theory of superconductivity. ${ }^{3}$ A two temperature state with hot electrons and relatively cold ions can be generated during fusion neutron irradiation, swift heavy ion impact, and ultra-short (femtosecond) laser pulse photoexcitation and typically lasts around $\tau_{e i}=1-100 \mathrm{ps}$.

In general, an accurate knowledge of $G_{0}$ enables the interpretation of the femtosecond electron diffraction measurements, ${ }^{4,5}$ theoretical studies of the dynamics of laser ablation and melting, ${ }^{6}$ and photochemical processes, and puts an upper time bound on the dynamics of non-thermal phase transitions, such as the one discussed in Ref. 7. Our study focuses on tungsten as it is an important technological material, which plays a central role in fusion power reactor development projects, such as ITER and ASDEX. Determining $G_{0}$ in W would enable more accurate simulations of primary radiation damage that incorporate the effects of electronic excitations, ${ }^{8}$ such as bombardment of a surface by $\mathrm{keV}$ ions and $\mathrm{MeV}$ neutron radiation cascade simulations with continuum-atomistic $2 \mathrm{~T}$ schemes. ${ }^{9,10}$ In fact, it has been shown that the residual cascade damage in $\alpha-\mathrm{Fe}$ is highly sensitive to the choice of $G_{0},{ }^{10}$ and a similar effect is expected in $\mathrm{W}$.

The e-p coupling constant, $G_{0}$, for metals can be calculated ab initio (see Refs. 11-13 for exemplar studies), however, despite the importance of the constant, such calculations are still rare. Indeed, there are no $a b$ initio calculations of $G_{0}$ for W. In addition, there is large uncertainty in the experimental values, ${ }^{14,15}$ based on measurements of transient reflectivity of a laser-excited solid, as these vary by almost an order of magnitude.

In this Letter, we present a combined state of the art experimental and theoretical determination of the electronphonon coupling constant in tungsten. We verify our first principles prediction of $G_{0}$ by analysis of pump-probe reflectivity measurement for a (100) W surface.
From a theoretical standpoint, the electron-phonon coupling strength in metals is commonly discussed via the value of $\lambda$ (the e-p coupling constant), or $G_{0}$ (the effective e-p coupling constant). Both of these can be obtained via the measurable Eliashberg spectral function ${ }^{11,16} D(\omega)$, combining the electron and phonon densities of states and the coupling matrix. This function can be directly calculated using different $a b$ initio codes. The e-p coupling constant is defined as: ${ }^{14,16}$

$$
\lambda=2 \int_{0}^{\infty} d \omega \times \frac{D(\omega)}{\omega},
$$

and the effective e-p coupling constant as: ${ }^{14,16,17}$

$$
G_{0}=\frac{1}{V_{c}}\left(\frac{\pi k_{B}}{\hbar}\right) \times \lambda\left\langle\omega^{2}\right\rangle \times g\left(\varepsilon_{F}\right),
$$

where $k_{B}$ is the Boltzmann's constant, $\hbar$ the reduced Planck's constant, $V_{c}$ the volume of the unit cell, $g\left(\varepsilon_{F}\right)$ the electronic density of states (e-DOS) at the Fermi level. The second moment of the Eliashberg function, $\lambda\left\langle\omega^{2}\right\rangle$, is given by

$$
\lambda\left\langle\omega^{2}\right\rangle=2 \int_{0}^{\infty} d(\hbar \omega) \times(\hbar \omega) \times D(\omega) .
$$

We note that Eq. (2) is justified for low electronic temperatures or at all temperatures where the e-DOS is only weakly energy dependent. ${ }^{11}$

To evaluate the Eliashberg function for tungsten in its ground state from ab initio calculations, we used the ABINIT plane wave density functional theory (DFT) code .$^{18,19}$ We use a $24 \times 24 \times 24 \boldsymbol{k}$-points grid, a $8 \times 8 \times 8$ $\boldsymbol{q}$-points grid, and $60 \mathrm{Ha}$ cutoff energy. The obtained $\lambda=0.28$ and $\lambda\left\langle\omega^{2}\right\rangle=108 \mathrm{meV}^{2}$ are both in good agreement with previous theoretical estimates. ${ }^{3,16,20}$ Combining this result with the calculated e-DOS obtained with the same set of parameters through Eq. (2), we obtain the effective electron-phonon coupling constant of $G_{0}=1.65 \times 10^{17} \mathrm{~W}$ $\mathrm{m}^{-3} \mathrm{~K}^{-1}$. 
We compare this theoretical prediction with the analysis of the results of the e-p coupling measurements using pumpprobe femtosecond spectroscopy, where an initial pulse (pump) which excites the electrons is followed by a delayed series of probe pulses. The analysis of experimental measurements of transient reflectivity of a laser-excited solid is based on the two-temperature model ${ }^{1,2}$ briefly described below.

The $2 \mathrm{~T}$ model $(2 \mathrm{TM})$ assumes a temporal hierarchy of the different relaxation processes-namely, a rapid thermalisation of electrons $\left(\tau_{e e} \sim 100 \mathrm{fs}\right.$ for most metals ${ }^{21}$ ), following laser excitation that occurs much quicker than the electron-ion equilibration. The 2TM (and its later variations for semiconductors ${ }^{22}$ and magnetic materials ${ }^{23}$ ) further assumes that distinct temperatures can be defined in the electronic $\left(T_{e}\right)$ and ionic subsystems $\left(T_{i}\right)$ and that these are governed by two separate coupled diffusion equations linked by the energy transfer proportional to the effective electronphonon coupling, $G$, i.e.,

$$
\begin{gathered}
C_{e}\left(T_{e}\right) \frac{\partial T_{e}}{\partial t}-\nabla \cdot\left(\kappa_{e} \nabla T_{e}\right)=-G\left(T_{e}-T_{i}\right)+S(z, t), \\
C_{i}\left(T_{i}\right) \frac{\partial T_{i}}{\partial t}-\nabla \cdot\left(\kappa_{i} \nabla T_{i}\right)=G\left(T_{e}-T_{i}\right) .
\end{gathered}
$$

Here, $C_{e, i}$ denote heat capacities and $\kappa_{e, i}$ thermal conductivities for the electronic and ionic systems, respectively. The constant $G$ in the $2 \mathrm{~T}$ model $^{1}$ is, by construction, the same parameter as in the Allen's formulation (i.e., Eq. (2)) in ground state at $T_{e}=300 \mathrm{~K} .{ }^{5,16,17}$ The $T_{e}$ dependence of $G$ for the range of $T_{e}$ achieved with different levels of laser excitations considered here is very weak. It is nonetheless included in the $2 \mathrm{~T}$ model for completeness: $G\left(T_{e}\right)=G_{0} \times f\left(T_{e}\right)$, where we have calculated $f\left(T_{e}\right)$ according to the method described in Refs. 17 and 24. Finally, $S$ represents the laser source term and is defined later through Equation (7).

As shown in Ref. 14, by knowing $T_{e}$ versus the time delay $(t)$ from the $2 \mathrm{~T}$ model, one can determine the e-p coupling with no free parameters. However, this is based on several assumptions: (i) The effects of electron and heat diffusion can be neglected, or are well-known. This is usually the case for a thin film, where the optical penetration depth (or ballistic electron range in the case of s- and d-block metals) is greater than the film's thickness. (ii) The reflectivity of a material can be well parameterised with respect to $T_{e}$ and $T_{i}$, either theoretically ${ }^{25}$ or experimentally. ${ }^{14,26}$ (iii) The electronic and ionic specific heat capacities in the 2TM are known.

Alternative experimental methods of e-p coupling constant determination are based on tunnelling (for superconductors), heat-capacity or transient angle-resolved photoemission spectroscopy (ARPES) measurements, or on indirect estimates from fits of the experimental damage threshold across laser pulse widths to the 2TM solutions. Here, we use an ab initio parameterised 2TM to verify our theoretical predictions of $G_{0}$ by analysing experimental reflectivity measurements of laser-excited tungsten.

In our experimental setup, a single-crystal W of $L=1 \mathrm{~mm}$ thickness at $300 \mathrm{~K}$ was excited by $\lambda_{p}=400 \mathrm{~nm} t_{p}=90 \mathrm{fs}$ (fwhm) laser pulse at various intensities. The optical reflection changes $\left(R(t) / R_{0}\right)$ were measured as a function of time delay between pump and probe pulses. The probe light is the white light pulse generated by a sapphire plate by a part of the fundamental beam. The incident angle of the probe light is $2^{\circ}$ from the (100) surface normal, while the pump pulse excites the sample with an incident angle of $4^{\circ}$. Changes in reflection intensities at the probe wavelengths $\lambda_{\text {obs }}=450-1200 \mathrm{~nm}$ were detected using spectroscopic detectors. The incident fluences $\left(F_{\text {inc }}\right.$ ) of pump laser were changed from 0.209 to $5.22 \mathrm{~mJ} / \mathrm{cm}^{2}$. We use the experimentally determined reflectivity at $400 \mathrm{~nm}$ $(R=0.42)$ to arrive at the corresponding absorbed fluences $(F)$ of 0.121 to $3.03 \mathrm{~mJ} / \mathrm{cm}^{2}$.

In Fig. 1, temporal evolution of the reflection changes $\left(R(t) / R_{0}\right)$ at $\lambda_{\text {obs }}=875 \mathrm{~nm}(1.42 \mathrm{eV})$ and $1000 \mathrm{~nm}(1.24 \mathrm{eV})$ are shown, where the reflection intensities are enhanced by excitation. The rapid changes in the intensity within $1.5 \mathrm{ps}$ after excitation are followed by gradual decreases in the range of $1.5-5 \mathrm{ps}$. We have measured the reflectivity until 10-20 ps, after which $R(t) / R_{0}$ evolution becomes significantly slower. Within this timeframe, the reflectivity changes due to the elevated $T_{e}(t \sim 0-5 \mathrm{ps})$, and $T_{i}\left(t_{s} \sim 10 \mathrm{ps}\right)$ can be separated.

We use an iterative two-step analysis method to reproduce the measured $R(t) / R_{0}$ evolution and, thus, extract $G_{0}$. We first construct the reflectivity function at a particular probe wavelength, $\left.\Delta R\left(T_{e}, T_{i}\right)\right|_{\lambda_{\text {obs }}}$ from a subset of the experimental data, assuming a value for $G_{0}$ in the $2 \mathrm{~T}$ model. As the second step, we calculate $R(t) / R_{0}$ evolution from the $2 \mathrm{~T}$ model via the previously calculated $\left.\Delta R\left(T_{e}, T_{i}\right)\right|_{\lambda_{\text {obs }}}$ and compare it with the experimental measurements for $R(t) / R_{0}$. We repeat the procedure, adjusting $G_{0}$ and $t_{0}$ (time when the pump pulse maximum arrives on the sample surface), to find the best fit for $R(t) / R_{0}$ using the least-squares method across a time domain ( $0.1-4.0 \mathrm{ps})$. A similar fitting method has also been employed for the temporal reflectivity analysis in thin films, where the thermal conduction details can be neglected. ${ }^{14}$ In the case of bulk materials, the initial rapid $R(t) / R_{0}$ peak decay is caused, however, by both components: ${ }^{26}$ the electron-phonon coupling and the electronic energy dissipation into the depth of the sample, which is mainly related to the spatiotemporal dynamics of $T_{e}$ as the ions remain relatively cold.

More specifically, we achieve the parameterisation of $\left.\Delta R\left(T_{e}, T_{i}\right)\right|_{\lambda_{o b s}}=\left.\Delta R\left(T_{e}\right)\right|_{\lambda_{o b s}}+\left.\Delta R\left(T_{i}\right)\right|_{\lambda_{o b s}}$ by correlating the
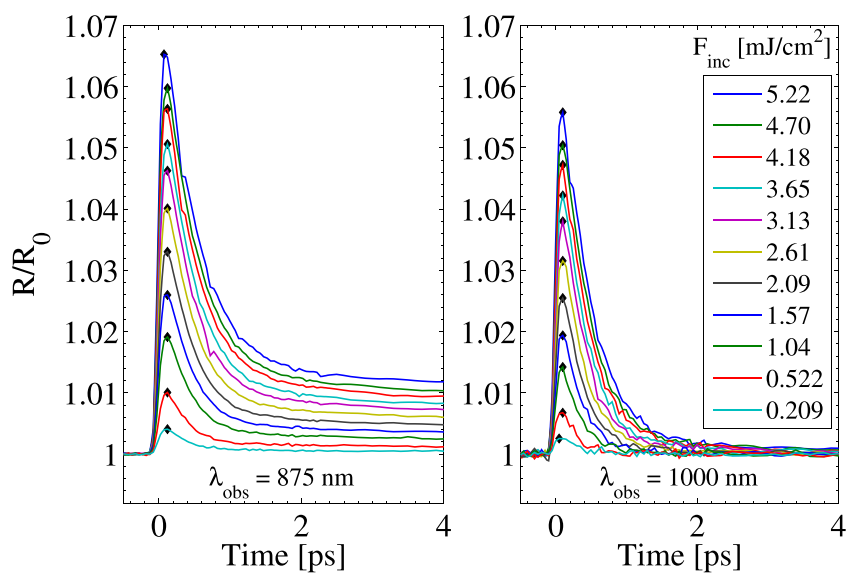

FIG. 1. Measured temporal evolution of reflectivity changes at $\lambda_{\text {obs }}=875 \mathrm{~nm}(1.42 \mathrm{eV})$ and $1000 \mathrm{~nm}(1.24 \mathrm{eV})$ for different incident laser fluences $\left(F_{\text {inc }}\right)$. Maxima are indicated with $(\bullet)$. 
peak of the reflectivity change $\left(\Delta R_{\max }\right)$ with the corresponding $T_{e}$ maxima from the $2 \mathrm{~T}$ calculations across different fluence measurements. We can assume that at this point $T_{i}$ is unperturbed, since the pump duration is extremely short $\left(t_{p}=90 \mathrm{fs}\right.$; also the initial peak $T_{e}$ is negligibly different when setting $G_{0}=0$ in the $2 \mathrm{~T}$ model). Similarly, $\Delta R\left(T_{i}\right)$ is correlated with $T_{i}$ calculated from the $2 \mathrm{~T}$ model at $t_{s}=10 \mathrm{ps}$, when the fast transient component related to $T_{e}$ has decayed away. An example of a $\Delta R\left(T_{e}, T_{i}\right)$ fit is presented in Fig. 2 . By construction, this iterative method always reproduces two reflectivity points-namely, the maximum and a point at selected $t_{s}$. However, the goodness of a fit for $R(t) / R_{0}$ is governed by $G_{0}$ and the details of the energy dissipation into the bulk. We note that we use only the highest incident fluence $\left(F_{\text {inc }}=5.22 \mathrm{~mJ} / \mathrm{cm}^{2}\right) R(t) / R_{0}$ data to fit $G_{0}$, having constructed $\Delta R\left(T_{e}, T_{i}\right)$ independently from the lower fluence data.

The technical details of 2TM are as follows. The electronic specific heat capacity is obtained from $a b$ initio using the method developed in Ref. 17 and $C_{i}$ from Ref. 27. The total thermal conductivity $\left(\kappa=\kappa_{e}+\kappa_{i}\right)$ at $T=300 \mathrm{~K}$ is $\kappa=174 \mathrm{~W} \mathrm{~m}^{-1} \mathrm{~K}^{-1}$ (Ref. 27) and $\kappa_{e} / \kappa=0.75$ (Ref. 28). We assume that $\kappa_{i}$ is independent of temperature, while $\kappa_{e}$ depends on both $T_{e}$ and $T_{i}$ according to the Drude model:

$$
\kappa_{e}\left(T_{e}, T_{i}\right)=\kappa_{e 0} \times \frac{C_{e}\left(T_{e}\right)}{C_{e}\left(T_{0}\right)} \times\left(T_{0} / T_{i}\right)^{-n},
$$

where $n=1.5$ has been fitted to reproduce the decrease of $\kappa$ as a function of the equilibrium (i.e. $T_{e}=T_{i}$ ) temperature, $T$, from $T=300 \mathrm{~K}$ to $2500 \mathrm{~K}$ from data in Ref. 27. Subsequent parameter sensitivity tests have shown that the $2 \mathrm{~T}$ model does not depend critically on the choice of $n$. The $\frac{C_{e}\left(T_{e}\right)}{C_{e}\left(T_{0}\right)}$ term dominates the $\kappa_{e}\left(T_{e}, T_{i}\right)$ variation since the changes in $T_{e}$ are much greater than in $T_{i} . S(z, t)$, the laser source term (pump), is assumed to be a Gaussian distribution in time $t$ with fwhm, $t_{p}=90 \mathrm{fs}$ and exponentially attenuated in the $z$ direction, i.e.,
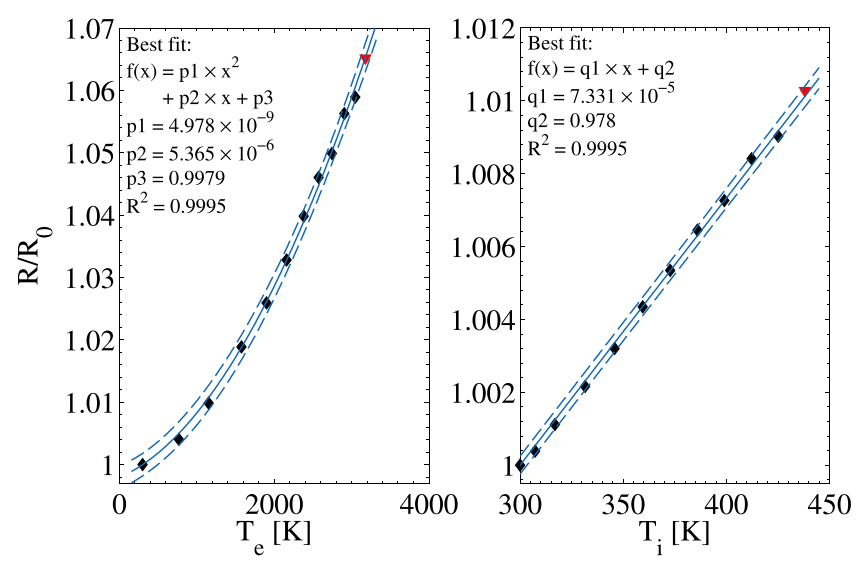

FIG. 2. Calculated reflectivity change $R(t) / R_{0}$ dependence on $T_{e}$ and $T_{i}$ for $\lambda_{\text {obs }}=875 \mathrm{~nm} .95 \%$ prediction bounds and lines of best fit are indicated as dashed and solid lines, respectively. Electronic temperature $\left(T_{e}\right)$ was obtained from the $2 \mathrm{~T}$ model and correlated with $R(t) / R_{0}$ maxima from experiment (small $T_{i}$ contribution), while the $T_{i}$ was correlated with $R(t) / R_{0}$ at time $t_{s}=10 \mathrm{ps}$ (small $T_{e}$ contribution). Points excluded from the fit are marked with $(\mathbf{\nabla})$.

$$
S(z, t)=\frac{2 F}{l_{p} t_{p}} \sqrt{\frac{\ln 2}{\pi}} \exp \left(-4 \ln 2\left(t-t_{0}\right)^{2} / t_{p}^{2}\right) \times \exp \left(-z / l_{p}\right) .
$$

The penetration depth in tungsten, $l_{p}=12.5 \mathrm{~nm}$, is obtained from the imaginary part of the index of refraction (i.e., extinction coefficient), $k=2.54$ (Ref. 29), at $\lambda_{p}=400 \mathrm{~nm}$. The optical reflectivity is calculated using $T_{e}$ and $T_{i}$ from the $2 \mathrm{~T}$ model averaged over the penetrations depths for the different probe wavelengths of $\lambda_{o b s}=875$ and $1000 \mathrm{~nm}$ ( $l_{p}=23.4$ and $23.7 \mathrm{~nm}$, respectively). The ballistic range of electrons is not included, as in transition metals it is of the same order or smaller than the penetration depth. ${ }^{30}$

We solve the $2 \mathrm{~T}$ model in continuum using an explicit Euler's scheme ${ }^{31}$ in one dimension and assume no lateral energy redistribution as the pump laser spot size was much larger than the probe. The length of the sample is $L=600 \mathrm{~nm}$ with $T_{0}$ Dirichlet boundary condition at $z=L$ for both $T_{e}$ and $T_{i}$. We checked that the choice of boundary conditions and $L$ does not change the results significantly. We took space-time steps of $\Delta z=2.5 \mathrm{~nm}$ and $\Delta t=0.4 \mathrm{fs}$, respectively. The simulations are typically run for $t=10 \mathrm{ps}$, past which $R(t) / R_{0}$ temporal changes are minimal and $T_{e}$ and $T_{i}$ equilibrate.

Fitting the 2TM self-consistently for two probe wavelengths treating the electron-phonon coupling as a free parameter, we obtain $G_{0}=1.4(3) \times 10^{17} \mathrm{~W} \mathrm{~m}^{-3} \mathrm{~K}^{-1}$, which is in a very good agreement with our theoretical prediction. The error stems from the uncertainty in the $\Delta R\left(T_{e}, T_{i}\right)$ parameterisation and a variation between the two $\lambda_{o b s}$ results. A set of example $R(t) / R_{0}$ fits with varying $G_{0}$ combining the 2TM analysis and experiment is presented in Fig. 3.

We note that the $G_{0}$ value for W obtained here is slightly smaller than the value of $2.0 \times 10^{17} \mathrm{~W} \mathrm{~m}^{-3} \mathrm{~K}^{-1}$, obtained by combining experimental and theoretical optical reflectivity data for thin films ${ }^{14}$ and much smaller than the experimentally determined $G_{0}=5-10 \times 10^{17} \mathrm{~W} \mathrm{~m}^{-3} \mathrm{~K}^{-1}$ (Ref. 15). However, the latter experiment was performed for bulk W,
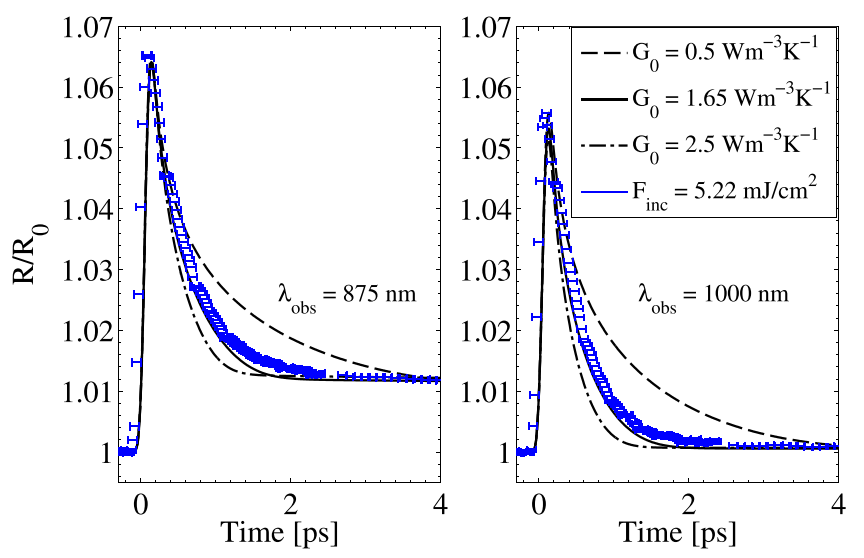

FIG. 3. 2T model (including electronic energy diffusion into the bulk and e-p coupling effects) predictions of temporal evolution of $R(t) / R_{0}$ against the experimental data for $\lambda_{o b s}=875 \mathrm{~nm}$ (left panel) and $1000 \mathrm{~nm}$ (right) for varying $G_{0}$. A fit using the ab initio e-p coupling $\left(G_{0}=1.65 \times 10^{17} \mathrm{~W} \mathrm{~m}^{-3}\right.$ $\mathrm{K}^{-1}$ ) in the $2 \mathrm{~T}$ model is shown. The best $2 \mathrm{~T}$ model fit to experimental data for both probe wavelengths is obtained with $G_{0}=1.4(3) \times 10^{17} \mathrm{~W} \mathrm{~m}^{-3} \mathrm{~K}^{-1}$ (not shown) using least-squares fit on the $t=0.1-4.0 \mathrm{ps}$ time domain. The experimental temporal resolution of the probe is indicated by horizontal error bars of width of $t=120 \mathrm{fs}$. 
where the electronic conduction was neglected, and, hence, the e-p value is very likely to be overestimated. This is in contrast with the results of Ref. 14, where all the samples were optically thin so that the pump-laser energy was distributed uniformly over the depth of the film. Furthermore, we note that the electronic energy diffusion into the sample can dramatically affect the reflectivity decay time and, hence, the electron-phonon coupling estimate from the 2TM in bulk samples. Our initial analysis neglecting the $T_{e}$ diffusion gave $G_{0} \sim 8.0 \times 10^{17} \mathrm{Wm}^{-3} \mathrm{~K}^{-1}$, a factor of five overestimation in line with the results of Ref. 15 for bulk W. We also observe that, while the $2 \mathrm{TM}$ reproduces the temporal decay of $R(t) / R_{0}$ very well, this decay is retarded by approximately $100 \mathrm{fs}$ with respect to $R_{\max }$. This may be either due to the experimental uncertainty, or may reflect a much weaker $G_{0}$ value for non-thermalised electrons, as suggested in Ref. 32.

In summary, we have determined the electron-phonon coupling constant in tungsten from ab initio calculations and high-resolution pump-probe reflectivity measurements analysed with the $2 \mathrm{~T}$ model. The very good agreement between the theory and experiment, adds a confidence in the $a b$ initio result of $G_{0}=1.65 \times 10^{17} \mathrm{~W} \mathrm{~m}^{-3} \mathrm{~K}^{-1}$. In addition, our results compare reasonably well with the previously reported value from a thin-film experiment. ${ }^{14}$

We conclude that for reflectivity measurements in bulk samples, the $T_{e}$ dissipation into the depth of the sample cannot be neglected even on a sub-picosecond timescale. However, once this effect is accounted for, the results of these measurements analysed with the 2TM give an accurate value for $G_{0}$. Both the reflectivity measurements analyzed with the 2TM and ab initio methods of calculating the e-p coupling constant can be readily applied to a wide range of bulk metals (which are considerably easier to prepare than thin films). The accurate determination of $G_{0}$ presented here will empower the multi-scale radiation damage and laser ablation in $\mathrm{W}$ studies leaving no adjustable parameters in most of the 2T-based models.

This work was supported by a specially promoted Research (No. 24000006) of Grant-in-Aid for Scientific Research from the JSPS, Japan. Via our membership of the UK's HPC Materials Chemistry Consortium, which is funded by EPSRC (EP/L000202), this work made use of the facilities of ARCHER, the UK's national high-performance computing service, which is funded by the Office of Science and Technology through EPSRC's High End Computing Programme.
${ }^{1}$ S. Anisimov, B. Kapeliovich, and T. Perel'man, Sov. Phys. JETP 39, 375 (1974).

${ }^{2}$ M. I. Kaganov, I. M. Lifshitz, and L. V. Tanatarov, Sov. Phys. JETP 4, 173 (1957).

${ }^{3}$ W. McMillan, Phys. Rev. 167, 331 (1968).

${ }^{4}$ Y. Giret, N. Naruse, S. L. Daraszewicz, Y. Murooka, J. Yang, D. M. Duffy, A. L. Shluger, and K. Tanimura, Appl. Phys. Lett. 103, 253107 (2013).

${ }^{5}$ Y. Giret, A. Gellé, and B. Arnaud, Phys. Rev. Lett. 106, 155503 (2011).

${ }^{6}$ L. V. Zhigilei, Z. Lin, and D. S. Ivanov, J. Phys. Chem. C 113, 11892 (2009).

${ }^{7}$ Y. Giret, S. L. Daraszewicz, D. M. Duffy, A. L. Shluger, and K. Tanimura, "Non-thermal solid-to-solid phase transitions in tungsten," Phys. Rev. B (submitted).

${ }^{8}$ D. M. Duffy and A. M. Rutherford, J. Nucl. Mater. 386-388, 19 (2009).

${ }^{9}$ D. M. Duffy and A. M. Rutherford, J. Phys.: Condens. Matter 19, 016207 (2007).

${ }^{10}$ A. M. Rutherford and D. M. Duffy, J. Phys.: Condens. Matter 19, 496201 (2007).

${ }^{11}$ B. Arnaud and Y. Giret, Phys. Rev. Lett. 110, 016405 (2013).

${ }^{12}$ A. Liu and A. Quong, Phys. Rev. B 53, R7575 (1996).

${ }^{13}$ S. Y. Savrasov, Phys. Rev. B 54, 16487 (1996).

${ }^{14}$ S. D. Brorson, A. Kazeroonian, J. S. Moodera, D. W. Face, T. K. Cheng, E. P. Ippen, M. S. Dresselhaus, and G. Dresselhaus, Phys. Rev. Lett. 64, 2172 (1990).

${ }^{15}$ J. Fujimoto, J. Liu, E. Ippen, and N. Bloembergen, Phys. Rev. Lett. 53, 1837 (1984).

${ }^{16}$ P. B. Allen, Phys. Rev. Lett. 59, 1460 (1987).

${ }^{17}$ Z. Lin, L. Zhigilei, and V. Celli, Phys. Rev. B 77, 075133 (2008).

${ }^{18}$ X. Gonze, G.-M. Rignanese, M. Verstraete, J.-M. Beuken, Y. Pouillon, R. Caracas, F. Jollet, M. Torrent, G. Zerah, M. Mikami, P. Ghosez, M. Veithen, J.-Y. Raty, V. Olevano, F. Bruneval, L. Reining, R. Godby, G. Onida, D. Hamann, and D. Allan, Z. Kristallogr. 220, 558 (2005).

${ }^{19}$ X. Gonze, B. Amadon, P.-M. Anglade, J.-M. Beuken, F. Bottin, P. Boulanger, F. Bruneval, D. Caliste, R. Caracas, M. Côté, T. Deutsch, L. Genovese, P. Ghosez, M. Giantomassi, S. Goedecker, D. Hamann, P. Hermet, F. Jollet, G. Jomard, S. Leroux, M. Mancini, S. Mazevet, M. Oliveira, G. Onida, Y. Pouillon, T. Rangel, G.-M. Rignanese, D. Sangalli, R. Shaltaf, M. Torrent, M. Verstraete, G. Zerah, and J. Zwanziger, Comput. Phys. Commun. 180, 2582 (2009).

${ }^{20}$ W. Butler and R. Williams, Phys. Rev. B 18, 6483 (1978).

${ }^{21}$ P. Musumeci, J. T. Moody, C. M. Scoby, M. S. Gutierrez, and M. Westfall, Appl. Phys. Lett. 97, 063502 (2010).

${ }^{22}$ H. M. van Driel, Phys. Rev. B 35, 8166 (1987).

${ }^{23}$ P.-W. Ma and S. L. Dudarev, Phys. Rev. B 83, 134418 (2011).

${ }^{24}$ X. Wang, D. Riffe, Y.-S. Lee, and M. Downer, Phys. Rev. B 50, 8016 (1994).

${ }^{25}$ J. Hohlfeld, D. Grosenick, U. Conrad, and E. Matthias, Appl. Phys. A 60, 137 (1995).

${ }^{26}$ M. Bonn, D. Denzler, S. Funk, M. Wolf, S.-S. Wellershoff, and J. Hohlfeld, Phys. Rev. B 61, 1101 (2000).

${ }^{27}$ T. Bergman, A. Lavine, and F. Incropera, Fundamentals of Heat and Mass Transfer, 7th ed. (John Wiley \& Sons, 2011).

${ }^{28}$ P. L. Garrity, J. Appl. Phys. 109, 073701 (2011).

${ }^{29}$ A. D. Rakic, A. B. Djurišic, J. M. Elazar, and M. L. Majewski, Appl. Opt. 37, 5271 (1998).

${ }^{30}$ J. Hohlfeld, S.-S. Wellershoff, J. Gdde, U. Conrad, V. Jhnke, and E. Matthias, Chem. Phys. 251, 237 (2000).

${ }^{31}$ A. Tveito and R. Winther, Introduction to Partial Differential Equations: A Computational Approach, Texts in Applied Mathematics (Springer, 1998).

${ }^{32}$ B. Y. Mueller and B. Rethfeld, Phys. Rev. B 87, 035139 (2013). 